\title{
Correction to: How Common is Statin Use in the Oldest Old?
}

\author{
Wade Thompson $^{1}$ (1) $\cdot$ Anton Pottegård ${ }^{2} \cdot$ Jesper Bo Nielsen ${ }^{1} \cdot$ Peter Haastrup ${ }^{1}$. Dorte Ejg Jarbøl ${ }^{1}$
}

Published online: 3 October 2018

(c) Springer Nature Switzerland AG 2018

\section{Correction to: Drugs \& Aging https://doi.org/10.1007/s40266-018-0567-x}

In Sect. 4.3 "Factors Associated with Statin Use in the Oldest Old", the second paragraph contains errors that affect the interpretation of the studies discussed with respect to the effect of co-morbidities.

The paragraph currently reads:

In the outpatient population, Chokshi et al. reported that a higher co-morbidity index was associated with a lower chance of receiving a statin [15]. This is in line with the findings of Noaman et al., who reported that a higher comorbidity index increased the likelihood of receiving a statin [26]. In contrast, Gnjidic et al. found that those with a higher co-morbidity index were more likely to receive a statin in a nursing home population [14]. Further, Tija et al. studied the advanced dementia population living in a nursing home and reported that statin use was more likely in patients with comorbidities such as hypertension, diabetes mellitus, or stroke [24]. These findings suggest that the presence of multiple co-morbidities decreases the likelihood of receiving a statin in the community but increases the likelihood in persons living in nursing homes. One explanation for the discrepancy is that in the Gnjidic et al. study, $94 \%$ of statin users were receiving statins for secondary prevention at baseline [14]. Thus, even with more co-morbidities, the validity of statin use may be perceived to be greater and people would be kept on statins.

It should read:

In the outpatient population, Chokshi et al. reported that a higher co-morbidity index was associated with a lower chance of receiving a statin [15], while Noaman et al. reported that a higher co-morbidity index increased likelihood of receiving a statin on hospital admission [26]. Gnjidic et al. found that those with a higher co-morbidity index were more likely to receive a statin in a nursing home population [14]. Furthermore, Tija et al. studied the advanced dementia population living in a nursing home and reported that statin use was more likely in patients with co-morbidities such as hypertension, diabetes, or stroke [24]. These findings suggest conflicting evidence for the effect of co-morbidities on statin use in the community, while increasing co-morbidity appears to raise the likelihood of receiving a statin for persons living in nursing homes. One explanation is that in the Gnjidic et al. study, $94 \%$ of statin users were receiving statins for secondary prevention at baseline [14]. Thus, even with more co-morbidities, the validity of statin use may be perceived to be greater and people would be kept on statins.

The original article can be found online at https://doi.org/10.1007/ s40266-018-0567-x.

Wade Thompson

wthomp01@gmail.com

1 Research Unit of General Practice, Department of Public Health, University of Southern Denmark, J.B. Winsløws Vej 9A, 5000 Odense C, Denmark

2 Research Unit of Clinical Pharmacology and Pharmacy, Department of Public Health, University of Southern Denmark, Odense, Denmark 